IRA-International Journal of Technology \& Engineering

ISSN 2455-4480; Vol.04, Issue 01 (2016)

Institute of Research Advances

http://research-advances.org/index.php/IRAJTE

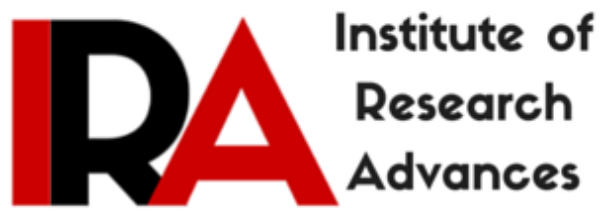

\title{
Analysis of optimal controlling system using Chebyshev Wavelets
}

\author{
V. Ramalakshmi ${ }^{1}$, T. Balasubramanian ${ }^{2}$, B.Ramesh Kumar ${ }^{3}$ \\ ${ }^{1}$ National Engineering College, Kovilpatti, TamilNadu, India. \\ ${ }^{2}$ Kamaraj College, Thoothukudi, TamilNadu, India. \\ ${ }^{3}$ Sree Sowdambika College of Engineering, Aruppukotai Tamil Nadu, India.
}

DOI: http://dx.doi.org/10.21013/jte.v4.n1.p7

How to cite this paper:

Ramalakshmi, V., Balasubramanian, T., \& Kumar, B. (2016). Analysis of optimal controlling system using Chebyshev Wavelets. IRA-International Journal of Technology \& Engineering (ISSN 2455-4480), 4(1). doi:http://dx.doi.org/10.21013/jte.v4.n1.p7

(C) Institute of Research Advances

(c) EY-NC

This works is licensed under a Creative Commons Attribution-Non Commercial 4.0 International License subject to proper citation to the publication source of the work.

Disclaimer: The scholarly papers as reviewed and published by the Institute of Research Advances (IRA) are the views and opinions of their respective authors and are not the views or opinions of the IRA. The IRA disclaims of any harm or loss caused due to the published content to any party. 


\begin{abstract}
In this paper, we derive new explicit formula for the matrix derivatives of chebyshev polynomial third degree because of this kind of polynomial is an important tool for numerical analysis of optimal controlling system. A numerical example is included to demonstrate the validity and applicability of the technique.
\end{abstract}

Keywords: Chebyshev wavelets, operational matrix of derivative, optimal control problem

\title{
1. Introduction:
}

In recent years, the study of ODEs and PDEs has attracted much attention due to an exact description of linear and nonlinear phenomena in fluid mechanics, viscoelasticity, biology, physics, engineering and other areas of science $[3,9,14,16]$. Wavelet theory is one of the most important tools for the optimal control system of the linear and nonlinear system of equation, wavelet techniques is utilized to solving the wide range of physical problems. The interest of this study is more accurate than integer-order models, that is, there are more degrees of freedom in the derivative order models are involved. Many researchers $[1,5,6,10,15]$ are stated that the various result in different kind of wavelet models ( Haar, Legendre, Galerkin, chebyshev etc.,). As particularly researcher has discussed chebyshev polynomial because of this polynomial is most suitable in numerical analysis including polynomial approximation, integral and differential equation $[2,7,8,11]$. The operational matrices of the derivatives have been determined for Chebyshev polynomials [7] and Legendre polynomials [4], and applied together with tau and pseudo spectral methods to solve some types of PDEs. The operational matrix of integration has been determined for several types of orthogonal polynomials, such as Chebyshev polynomials of the first kind [12], Legendre polynomials [13]. Recently, Singh et al. [17] derived the Bernstein operational matrix of integration. In this paper we derive new explicit formula for the matrix derivatives of chebyshev polynomial of third degree because of this kind of polynomial is an important application of the numerical analysis of optimal controlling system of the matrix derivatives. Integral and differential equation $[2,7,8,11]$ The operational matrices of the derivatives have been determined for Chebyshev polynomials [7] and Legendre polynomials [4], and applied together with tau and pseudo spectral methods to solve some types of PDEs. The operational matrix of integration has been determined for several types of orthogonal polynomials, such as Chebyshev polynomials of the first kind [12], Legendre polynomials [13]. Recently, Singh et al. [17] derived the Bernstein operational matrix of integration. In this paper we derive new explicit formula for the matrix derivatives of chebyshev polynomial of third degree because of this kind of polynomial is an important application of the numerical analysis of optimal controlling system of the matrix derivatives.

2. Chebyshev Wavelets: Wavelets constitute a family of functions constructed from dilation and translation of a single function $\psi(x)$ called the mother wavelet. When the dilation parameter "a" and the translation parameter " $b$ " varies continuously we have the following family of continuous wavelets as $\psi_{a, b}(x)=|a|^{-1 / 2} \psi\left(\frac{x-b}{a}\right), a, b \in R, a \neq 0$. If the parameters a and $\mathrm{b}$ are discrete value, where $a=a_{0}^{-k}, b=n b_{0} a_{0}^{-k}, a_{0}>1, b_{0}>0$. The family of the discrete wavelet as follows: $\psi_{k, n}(x)=|a|^{k / 2} \psi\left(a_{0}^{k} x-n b_{0}\right), k, n \in R$, where $\quad \psi_{k, n} \in L^{2}(R)$. Suppose that, here $a_{0}=2$ and $b=1$, the family of discrete wavelet forms as an orthonormal basis.

2.1 Second Degree of Chebyshev Wavelets: Four arguments are involving this kinds of wavelet, the family of wavelet is $\psi_{k, n}(x)=\psi(k, n, m, x)$, where $n=1,2,3 \ldots .2^{k}, k \in R^{+}, \mathrm{m}-$ 
denotes the degree of Chebyshev Polynomial, $x \in[0,1)$ The Second Degree of Chebyshev Wavelets is defined as $: \psi_{m, n}(x)=\left\{\begin{array}{ll}2^{k / 2} \bar{T}_{m}\left(2^{k} x-2 n+1\right), & \frac{n-1}{2^{k-1}} \leq x<\frac{n}{2^{k-1}} \\ 0 & \text { otherwise }\end{array}\right\}, \quad$ where $\bar{T}_{m}(x)=\sqrt{2 / \pi} T_{m}(x), \mathrm{m}=0,1,2 \ldots \mathrm{M}-1$. Here $\bar{T}_{m}(x)$ are the second degree of Chebyshev polynomials " $m$ " with respect to the weight function of $w(x)=\sqrt{1-x^{2}}$ on the interval $[-1,1]$ and satisfy the recursive formula: $T_{0}(x)=1, T_{1}(x)=2 x \ldots . . T_{m+1}(x)=2 x T_{m}(x)-T_{m-1}(x), \mathrm{m}=1,2,3$..

\subsection{Third degree of Chebyshev Wavelets:}

Four arguments are involving this kinds of wavelet, the family of wavelet is $\psi_{k, n}^{3}(x)=\psi^{3}(k, n, m, x)$, where $n=1,2,3 \ldots .2^{k}, k \in R^{+}, \mathrm{m}-$ denotes the degree of Chebyshev Polynomial, $x \in[0,1)$. The third Degree of Chebyshev Wavelets is defined as [14]

$\psi_{m, n}(x)=\left\{\begin{array}{ll}2^{k / 2} \bar{T}_{m}\left(2^{k+1} x-2 n+1\right), & \frac{n-1}{2^{k}} \leq x<\frac{n}{2^{k}} \\ 0 & \text { otherwise }\end{array}\right\}$----(2) where $\bar{T}_{m}(x)=\sqrt{1 / \pi} T_{m}(x)$, Here $\bar{T}_{m}(x)$ are the third degree of Chebyshev polynomials " $\mathrm{m}$ " with respect to the weight function of $w(x)=\sqrt{\frac{1+x^{2}}{1-x}}$ on the interval $[-1,1]$ and satisfy the recursive formula [13], $T_{0}(x)=1, T_{1}(x)=2 x-1$ $, T_{2}(x)=4 x^{2}-2 x-1, T_{3}(x)=8 x^{3}-4 x^{2}-4 x+1 \ldots \ldots T_{m}(x)=2 x T_{m-1}(x)-T_{m-2}(x)$

To obtain the recurrence relation, we have

$T_{m}(\cos 2 \theta)=\frac{\cos (2 n+1) \theta}{\cos \theta}, T_{m+1}(\cos 2 \theta)=\frac{\cos (2 n+1) \theta \cos 2 \theta+\sin (2 n+1) \theta \sin 2 \theta}{\cos \theta}$,

$T_{m-1}(\cos 2 \theta)=\frac{\cos (2 n+1) \theta \cos 2 \theta-\sin (2 n+1) \theta \sin 2 \theta}{\cos \theta}$

The eight basis functions when $\mathrm{M}=3, \mathrm{k}=1$ are given by:

For the interval $0 \leq x<0.5$, we obtained the family of the Chebyshev wavelet polynomial is: $\psi_{1,0}^{3}=\sqrt{2 / \pi}, \quad \psi_{1,1}^{3}=\sqrt{2 / \pi}(8 x-3)$, $\psi_{1,2}^{3}=\sqrt{2 / \pi}\left((8 x-3)^{2}+(8 x-3)-1\right)$,

$\psi_{1,3}^{3}=\sqrt{2 / \pi}\left((8 x-3)^{3}+2(8 x-3)^{2}-(8 x-3)-1\right)$

For the interval $0.5 \leq x<1$, we obtained the family of the Chebyshev wavelet polynomial is: $\psi_{2,0}^{3}=\sqrt{2 / \pi}, \quad \psi_{2,1}^{3}=\sqrt{2 / \pi}(8 x-7), \quad \psi_{2,2}^{3}=\sqrt{2 / \pi}\left((8 x-7)^{2}+(8 x-7)-1\right)$, $\psi_{2,3}^{3}=\sqrt{2 / \pi}\left((8 x-7)^{3}+2(8 x-7)^{2}-(8 x-7)-1\right)$

A function $\mathrm{f}(\mathrm{x})$ expanded as the interval $[0,1)$ as follows: $f(x)=\sum_{n=1}^{\infty} \sum_{m=0}^{\infty} f_{n, m} \psi_{n, m}^{3}(x)$-----(5) 
Where $f_{n, m}=\left(f(x), \psi_{n, m}^{3}(x)\right)$, The infinite series (5) is truncated and it can be written as: $f(x)=\sum_{n=1}^{2^{k}} \sum_{m=0}^{M-1} f_{n, m} \psi_{n, m}^{3}(x)=F^{(x)} \psi^{3}(x)$------- (6), where $\mathrm{F}$ and $\psi^{3}(x)$ are $2^{k}(M \times 1)$ Matrices are given by:

$$
\begin{aligned}
& F=\left[f_{1,0}, f_{1,1}, f_{1,2}, \ldots . f_{1, M-1}, f_{2,0}, f_{2,1} \ldots \ldots . . f_{2, M-1}, \ldots . f_{2^{k}, 0}, f_{2^{k}, 1}, \ldots \ldots f_{2^{k}, M-1}\right. \\
& \psi^{3}(x)=\left[\psi^{3}{ }_{1,0}(x), \psi^{3}{ }_{1,1}(x), \psi^{3}{ }_{1,2}(x), \ldots . \psi^{3}{ }_{1, M-1}(x),\right. \\
& \left.\psi^{3}{ }_{2,0}(x), \psi^{3}{ }_{2,1}(x) \ldots \ldots . . \psi^{3}{ }_{2, M-1}(x), \ldots . \psi^{3}{ }_{2^{k}, 0}(x), \psi^{3}{ }_{2^{k}, 1}(x), \ldots \ldots . \psi_{2^{k}, M-1}^{3}(x)\right]^{T}
\end{aligned}
$$

\section{Proposed the operational of matrix derivative:}

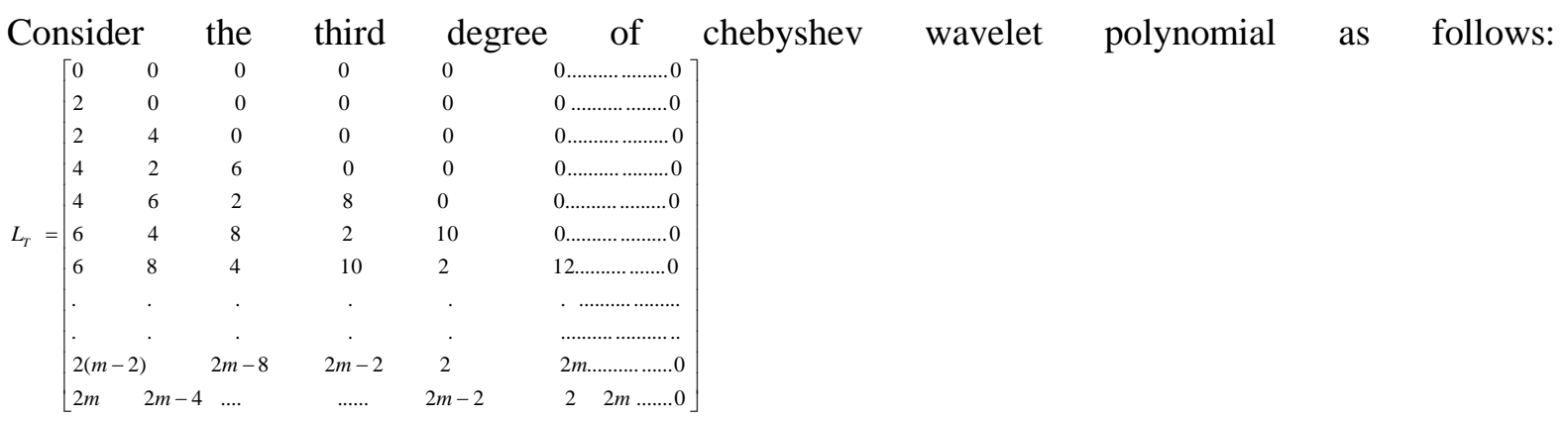

Where $\mathrm{L}$ denotes the differential operator of $\mathrm{x}, \mathrm{m}$-denotes the degree of the chebyshev wavelet polynomial. If $m$ is odd then $L_{T}$ becomes $\frac{d_{T_{m}}}{d x}=\left[\sum_{s=\text { even }}^{m-1}(m+s+1) T_{s}+\sum_{s=o d d}^{m-2}(m-s) T_{s}\right]--(8 \mathrm{a})$

Suppose that, if $m$ even then $L_{T}$ becomes $\frac{d_{T_{m}}}{d x}=\left[\sum_{s=\text { even }}^{m-2}(m-s) T_{s}+\sum_{s=d d}^{m-1}(m+s+1) T_{s}\right]$

In matrix form, the relations (8) are $T_{m}=L_{T} T(x)$

Where $L_{T}$ is an $(\mathrm{m}+1) \times(\mathrm{m}+1)$ matrix and $T(x)$ is the vector of Chebyshev polynomial of the third degree chebyshev polynomial is $T(x)=\left[T_{0}, T_{1}, T_{2}, \ldots . . T_{m}\right]^{T}$

The following Lemma is the new relation about the derivative of third degree chebyshev wavelets operational matrix.

3.1 Lemma: The new relation about the first derivative of the third degree chebyshev wavelet is $\psi^{3}(x)=L_{\psi^{s}} \psi^{3}(x)-------(11)$, where $\psi^{3}$ is the vector space of the chebyshev wavelet in (7) and $L_{\psi^{s}}$ is the $2^{k-1}((M+1) \times(M+1))$ operational matrix of third degree Chebyshev wavelets as follows: $L_{\psi^{s}}=D(z)$, where D-denotes the diagonlation of the matrix and z-denotes $((M+1) \times(M+1))$ matrix and the element $(\mathrm{p}, \mathrm{s})$ defined as: 
if $\mathrm{m}$ is odd $z_{p, s}$ becomes $Z_{p, s}=2^{k+1}\left[\sum_{s=\text { even }}^{p-1}(p+s+1) T_{s}+\sum_{s=o d d}^{p-2}(p-s) T_{s}\right]$

if $\mathrm{m}$ is even $z_{p, s}$ becomes $Z_{p, s}=2^{k+1}\left[\sum_{s=\text { even }}^{p-2}(p-s) T_{s}+\sum_{s=o d d}^{p-1}(p+s+1) T_{s}\right]$

\section{Proof:}

In (2), the family of chebyshev wavelet can be written as

$\psi_{p}^{3}(x)=\left\{\begin{array}{ll}2^{k / 2} \sqrt{2 / \pi} \bar{T}_{p}\left(2^{k+1}(x)-n\right. & x \in\left[\frac{n}{2^{k}}, \frac{n-1}{2^{k}}\right] \\ 0 & \text { otherwise }\end{array}\right\}$

where $p=0,1,2 \ldots .\left(2^{k} M\right)+1, m=0,1,2, \ldots . M$ and $n=0,1,2 \ldots . .2^{k}-1$

From(2), differenciating with respect to $\mathrm{x}$, we get

$L\left(\psi_{p}^{3}(x)\right)=\left\{\begin{array}{ll}2^{k / 2} \sqrt{2 / \pi} 2^{k} \bar{T}_{m}\left(2^{k+1}(x)-n\right) & \frac{n}{2^{k}} \leq x<\frac{n+1}{2^{k}} \\ 0 & \text { otherwise }\end{array}\right\}$

Therefore, from the equation (2) and (13) obtained equation (15):

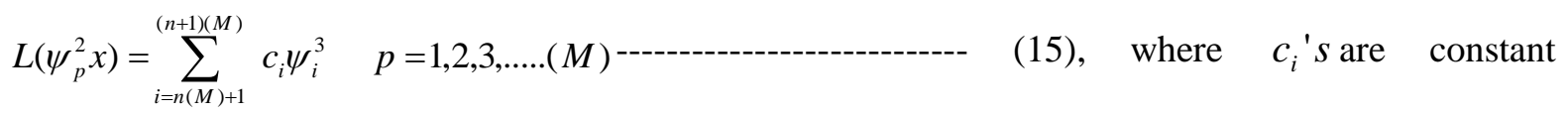
coefficients and we obtained $L\left(\psi_{p}^{2} x\right)=0 \quad p=0,(M+1), \ldots \ldots .2^{k-1}(M+1)$

Finally equation (14) become zero, because $L\left(T_{0}^{2} x\right)=0 \quad$, consequently the first row of the matrix $p$ is zero. Using the relation from (8) and (14) leads to equation (12). We obtained the require result.

4. Numerical Example: Consider the finite time quadratic problem $I=\int_{0}^{1} y^{2} d x$, The exact solution of this problem is given by

$$
y_{1}(x)=x^{3}-3 x^{2}+x+1 \text { and } y=3 x-6
$$

First, we find the Hamiltonian Equation: $H=y^{2}+\lambda_{1} x_{2}+\lambda_{2} y$, where $\lambda_{1}=\frac{-\partial H}{\partial x_{1}}$ and $\lambda_{2}=\frac{-\partial H}{\partial x_{2}}$ and $0=\frac{\partial H}{\partial y}$, From the above equations, we obtain the following

$\frac{d y}{d x}+\frac{d^{2} y}{d x^{2}}=3 x^{2}-5$

Now $\mathrm{y}(\mathrm{x})$ approximated with the third degree of chebyshev wavelets with $\mathrm{M}=4$ and $\mathrm{K}=1$

i.e) $x=c^{T} \psi^{3}$, (16) becomes $c^{T}\left(D_{\psi^{3}}\right) \psi^{3}(x)=d^{T} \psi^{3}(x)$, where $d^{T} \psi^{3}(x)=3 x^{2}-5$---- (17) 
$\operatorname{But} D_{\psi_{3 \times 3}^{3}}=\left[\begin{array}{cccc}R & -- & -- & O \\ - & -- & -- & -- \\ -- & -- & -- & -- \\ O & -- & -- & R\end{array}\right]$, where $R=\left[\begin{array}{cccc}0 & 0 & 0 & 0 \\ 2 & 0 & 0 & 0 \\ 2 & 4 & 0 & 0 \\ 4 & 2 & 6 & 0\end{array}\right]$

From (17), we obtained the system of equation is:

$2 c_{2}+34 c_{3}+68 c_{4}=-1.419,4 c_{3}+98 c_{4}=0.0734,6 c_{4}=0.0146, c_{8}=0.014$

$2 c_{6}+34 c_{7}+68 c_{8}=-0.832,4 c_{7}+98 c_{8}=0.190$

and $d=\left[\begin{array}{llllll}-1.419 & 0.0734 & 0.0146 & -0.832 & 0.190 & 0.014\end{array}\right]^{T}$

The boundary condition of the equation is given by:

$y(0)=c^{T} \psi^{3}(0)=1$ and $y(1)=c^{T} \psi^{3}(0)=0$

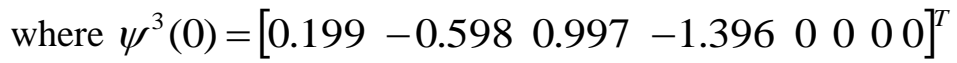

$$
\psi^{3}(1)=\left[\begin{array}{llllllll}
0 & 0 & 0 & 0 & 0.199 & 0.199 & 0.199 & 0.199
\end{array}\right]^{T}
$$

Solving the (18\&19) we get the values of $c^{T}$

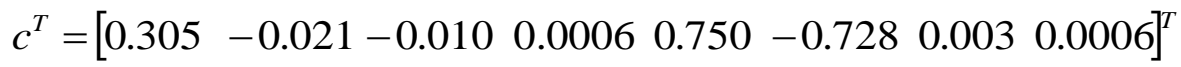

Table: 1 The following table represents the Multiple Level DWT Optimal value (Frequency Status) and to analyze the Accurate Approximation Frequency is:

\begin{tabular}{|l|l|l|l|l|}
\hline $\begin{array}{l}\text { Frequency } \\
\text { Range (x) }\end{array}$ & $\begin{array}{l}\text { Accurate } \\
\text { Frequency } \\
\text { Solution } \\
(\mathrm{y}(\mathrm{x}))\end{array}$ & $\begin{array}{l}\text { Approximate } \\
\text { Frequency } \\
\text { Solution } \\
(\mathrm{M}=4)\end{array}$ & Error Frequency & $\begin{array}{l}\text { Accurate } \\
\text { Approximation } \\
\text { Frequency }\end{array}$ \\
\hline 0 & 1 & 1 & 0.000 & 0.9859 \\
\hline 0.1 & 0.267 & 0.218 & 0.059 & 0.3776 \\
\hline 0.2 & 0.27 & 0.27 & 0.000 & 0.3281 \\
\hline 0.3 & 0.264 & 0.24 & 0.020 & 0.2312 \\
\hline 0.4 & 0.246 & 0.246 & 0.000 & 0.4081 \\
\hline 0.5 & 0.218 & 0.218 & 0.000 & 0 \\
\hline 0.6 & 0.184 & 0.163 & 0.021 & 0 \\
\hline 0.7 & 0.143 & 0.142 & 0.001 & 0 \\
\hline 0.8 & 0.098 & 0.098 & 0.000 & 0 \\
\hline 0.9 & 0.48 & 0.48 & 0.000 & 0 \\
\hline 1 & 0 & 0 & 0.000 & 0 \\
\hline
\end{tabular}

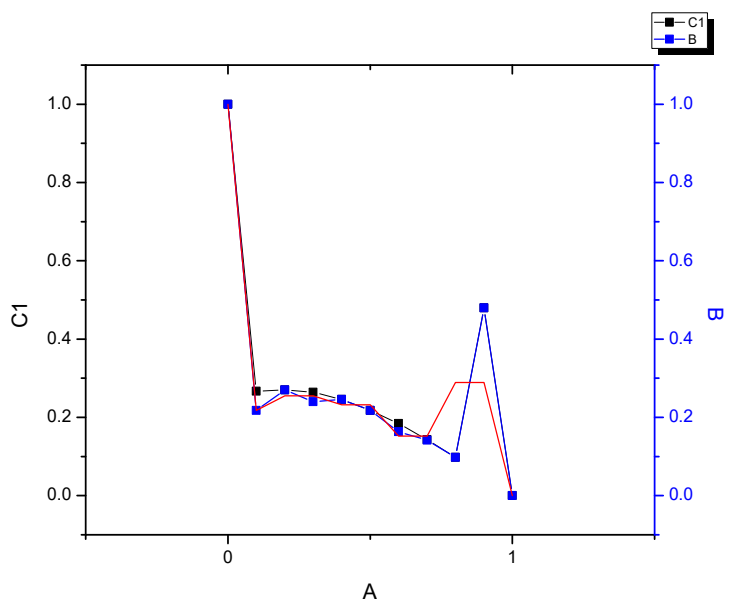


Fig : (i) Graphical Structure of the Frequency Level of DWT is

B - denotes the Accurate Frequency, C1-denotes the Approximation Frequency,

A-denotes the range of the Frequency level

\section{Conclusion:}

In this paper, our proposed algorithm obtains the numerical wavelet solution for matrix derivative of third order linear problems are analyzed and discussed the optimal frequency level for chebyshev polynomial. Our Methodology is most helpful to the optimal control system of linear equation. In this method we easily to analyze the system optimization level (error \& accurate frequency). The authors welcome suggestions for the improvement of the paper.

\section{References:}

[1] Babolian, E., Fattah Zadeh, F, 2007, "Numerical solution of differential equations by using Chebyshev wavelet operational matrix of integration". Appl. Math. Comput, Vol.188, pp. 417-426.

[2] Bhrawy, A.H., Alofi, A.S, 2013, "The operational matrix of fractional integration for shifted Chebyshev polynomials", Applied Mathematics Letters, Vol.26, pp. 25-31.

[3] Bhrawy, A.H., Alghamdi, A, 2012, "A Shifted Jacobi-Gauss-Lobatto Collocation Method for Solving Nonlinear Fractional Langevin Equation", Boundary Value Problems 62

[4] Bhrawy, A.H., Alofi, A.S and Ezz-Eldien, S.S, 2011, "A quadrature tau method for fractional differential equations with variable coefficients", Appl. Math. Lett, Vol. 24, pp. 2146-2152.

[5] Cattani, C., Kudreyko, A, 2010, "Harmonic wavelet method towards solution of the Fredholm type integral equations of the second kind", Appl. Math. Comp, Vol. 215, pp.4164-4171.

[6] Dehghan, M., Saadatmandi, A, 2008, "Chebyshev finite difference method for Fredhalm integrodifferential equation", Int. J. Comput. Math, Vol.85 (1), pp.123-130

[7] Doha, E.H., Bhrawy, A.H and Ezz-Eldien, S.S, 2011, "A Chebyshev spectral method based on operational matrix for initial and boundary value problems of fractional order", Comput. Math. Appl, Vol.62, pp. 2364-2373.

[8] Fox, Lslie and Ian Bax Parker, 1968, "Chebyshev Polynomials in Numerical Analysis", Oxford university press, London, vol.29.

[9] Kilbas, A.A., Srivastava, H.M and Trujillo, J.J, 2006, "Theory and Applications of Fractional Differential Equations", North-Holland Math. Stud., vol. 204, Elsevier Science B.V., Amsterdam,

[10] Mohammadi, F., Hosseini, M.M., 2010, "Legendre wavelet method for solving linear stiff systems". J. Adv. Res. Diff. Eqns, Vol. 2(1), pp. 47-57.

[11] Nkwanta, A., Barnes, E.R, 2012, "Two Catalan-type Riordan arrays and their connections to the Chebyshev polynomials of the first kind", Journal of Integer Sequences, Vol.15, pp. 1-19.

[12] Paraskevopoulos, P.N, 2013, "Chebyshev series approach to system identification, analysis and control", Journal of the Franklin Institute, Vol.316, pp. 135-157.

[13] Paraskevopoulos, P. N, 1985," Legendre series approach to identification and analysis of linear systems", IEEE Transactions Automatic Control, Vol.30, pp. 585-589.

[14] Podlubny, I, 1999, "Fractional Differential Equations", Math. Sci. Eng., Academic Press, New York,

[15] Rawashdeh, EA. 2011, "Legendre Wavelets Method for Fractional Integro-Differential Equations". Appl. Math. Sci., Vol.5, pp. 2465-2474.

[16] Samko, S.G., Kilbas, A.A., and Marichev, O.I, 1993, "Fractional Integrals and Derivatives. Theory and Applications", Gordon and Breach, Yverdon.

[17] Singh, A.K., Singh,V.K., and Singh, O.P, 2009, "The Bernstein operational matrix of integration", Appl.Math. Sciences, Vol.3, pp. 2427-2436. 